\title{
Prevalência de fluorose e concentração de fluoreto em água potável
}

Fluorosis prevalence and fluoride concentration in drinking water

Prevalencia de fluorosis y concentración de fluoruro en agua potable

Sabrina Gonçalves RIATTO ${ }^{1}$

Vitor Matheus da Silva LUNA ${ }^{2}$

${ }^{1}$ Professora Doutora, Faculdade de Odontologia, UNIESP Centro Universitário, 58109-303, Cabedelo - PB, Brasil

${ }^{2}$ Curso de Graduação em Odontologia, Faculdade de Odontologia, UNIESP Centro Universitário, 58109-303, Cabedelo - PB, Brasil

\section{Resumo}

Introdução: Na odontologia, o íon flúor, também conhecido como fluoreto, apresenta efeitos profiláticos e terapêuticos, porém pode trazer riscos. A sua utilização em águas de abastecimento público e em outros produtos deve ser entendida e controlada para minimizar efeitos indesejados às dentições, como a fluorose dentária. O monitoramento da fluorose em um país tem sua importância para comprovar se as cifras estão estabilizadas, uma vez que se conhece um crescente potencial imigratório mundial. Objetivo: Revisão da literatura dos últimos anos, buscando informações sobre a relação entre a concentração de fluoreto em água natural potável e a prevalência de fluorose em variadas populações de diferentes países. Materiais e Métodos: Buscaram-se publicações nas principais bases de dados: Scopus, Portal Periódicos Capes, Scielo, Biblioteca Virtual em Saúde, PubMed e Google Acadêmico, através das palavras-chave: Fluorose dentária, Flúor, Fluoreto, Epidemiologia e Água potável, nos idiomas português, espanhol e inglês. Dos 38 artigos selecionados, 29 foram utilizados no presente trabalho. Resultados: Dos países estudados, a Etiópia (com 12 partes por milhão de Flúor na água potável) obteve maior índice de fluorose dentária ( $100 \%$ da amostra), e o menor índice de fluorose foi encontrado na Síria (1.39 partes por milhão de Flúor na agua potável), equivalente a 15\%. Conclusão: Pôde-se concluir que a prevalência e a severidade da fluorose dentária geralmente são maiores nas populações que vivem em regiões com altas concentrações de fluoreto na água natural potável.

Descritores: Fluorose Dentária; Flúor; Fluoreto de Sódio; Epidemiologia; Água Potável.

\section{Abstract}

Introduction: In dentistry the fluorine ion - also known as fluoride - shows prophylactic and therapeutic effects but it can also present risks. Its use in public water supply and other products should be understood and controlled in order to minimize unwanted effects on dentitions, such as dental fluorosis. The monitoring of dental fluorosis in a country is important to verify whether figures are stabilized, once a growing global immigration potential is known. Objective: To carry out a literature review of the last years, searching for information about the relations between fluoride concentration in drinking water and the prevalence of fluorosis in various populations of different countries. Materials and Methods: Was sought publications in the main databases: Scopus, Portal Periódicos Capes, Scielo, Virtual Health Library, PubMed and Google Scholar, using the following keywords: Dental fluorosis, Fluorine, Fluoride, Epidemiology and Drinking Water, in Portuguese, Spanish and English. Of the 38 articles selected, 29 were used in this study. Results: Of the countries studied, Ethiopia (with 12 parts per million of fluorine in drinking water) obtained the highest index of dental fluorosis ( $100 \%$ of the sample) while the lowest fluorosis index was found in Syria (1.39 parts per million of fluorine in drinking water), equivalent to $15 \%$. Conclusion: It can be concluded from this study that the prevalence and severity of dental fluorosis are generally higher in populations living in regions with high concentrations of fluoride in drinking water, since it is considered the main natural source of fluorine ion in many communities.

Descriptors: Fluorosis, Dental; Fluorine; Sodium Fluoride; Epidemiology; Drinking Water.

\section{Resumen}

Introducción: En odontología, el ion flúor - también conocido como fluoruro - tiene efectos profilácticos y terapéuticos, pero puede presentar riesgos. Su uso en el agua de abastecimiento público y en otros productos debe ser entendido y controlado para minimizar los efectos no deseados en las denticiones, como la fluorosis dental. El monitoreo de la fluorosis dental en un país tiene su importancia para verificar si las cifras están estabilizadas, una vez que se conoce un potencial inmigratorio global en crecimiento. Objetivo: Revisar la literatura de los últimos años, buscando información sobre la relación entre la concentración de fluoruro en el agua potable y la prevalencia de fluorosis en distintas poblaciones de diferentes países. Materiales y Métodos: Se buscaron publicaciones en las bases de datos: Scopus, Portal Periódicos Capes, Scielo, Biblioteca Virtual en Salud, PubMed y Google Académico, mediante las palabras clave: Fluorosis dental, Fluoruro, Flúor, Epidemiología y Agua potable, en portugués, español e inglés. De los 38 artículos seleccionados, 29 fueron utilizados en este estudio. Resultados: De los países estudiados, Etiopía (12 partes por millón Flúor en agua potable) obtuvo el índice más alto de fluorosis dental (100\% de la muestra), mientras el índice de fluorosis más bajo se encontró en Siria (1.39 partes por millón Flúor en agua potable), equivalente a un $15 \%$. Conclusión: se pudo concluir en este estudio que la prevalencia y la gravedad de la fluorosis dental son generalmente más altas en las poblaciones que viven en regiones con altas concentraciones de fluoruro en el agua potable.

Descriptores: Fluorosis Dental; Flúor; Fluoruro de Sodio; Epidemiología; Agua Potable.

INTRODUÇÃO

O efeito físico-químico anticárie do fluoreto ocorre por meio da ação tópica e continuada nos elementos dentários. Isto significa que a sua incorporação ao dente durante a odontogênese tem menor significado, visto que não é necessário ingeri-lo para obter seus benefícios ${ }^{1}$.

A partir dessa informação, houve o uso tópico de fluoreto com a preocupação de prevenção e promoção de saúde, uma vez que se pode contar com estratégias acessíveis, eficazes e diversas de aplicação de fluoreto.
Os métodos atuais de administração de fluoreto podem ser classificados em métodos autoaplicáveis com destaque para os dentifrícios fluoretados, os métodos aplicados por profissionais da Odontologia e os métodos populacionais. No âmbito populacional, a fluoretação das águas de abastecimento público encabeça a lista dos programas de fluoretação comunitários, seguido pelas intervenções com o uso de sal de cozinha e leite fluoretados ${ }^{2}$.

Devido à acessibilidade e às referidas diversas formas de aplicação dos íons flúor, 
deve-se considerar a possibilidade de múltipla exposição com a associação de mais de uma forma de contato com a substância ${ }^{3}$, além de existir a preocupação com a ingestão excessiva da substância no período de desenvolvimento dentário (odontogênese), podendo desencadear um problema de saúde bucal conhecido como fluorose dentária ${ }^{1,4}$.

O conhecimento sobre 0 íon flúor mostra-se imprescindível, assim como seu uso profilático e terapêutico, seus benefícios e riscos, para um melhor resultado em saúde bucal, e a importância deste tema impulsionou esta revisão de literatura, que tem como objetivo buscar estudos sobre a relação entre a concentração de fluoreto na água natural e a prevalência de fluorose.

MATERIAL E MÉTODO

Os dados para a realização deste trabalho foram obtidos através de busca na literatura eletrônica nas seguintes bases de dados: Scopus, Portal Periódicos Capes, Scielo, BVS, PubMed e Google Acadêmico. Utilizaramse os seguintes descritores, e seus correspondentes nos idiomas inglês e espanhol: Fluorose Dentária, Flúor, Fluoreto de Sódio, Epidemiologia e Água Potável..

Foram incluídas publicações recentes; de qualquer país e língua, dando-se preferência para publicações em revistas com índice de impacto JCR e/ ou QUALIS. Foram excluídos estudos tais como teses, dissertações e monografias, embora foram analisadas as respectivas bibliografias como base para encontrar possíveis estudos de interesse.

Os artigos pré-selecionados pelos seus títulos, tiveram os seus resumos lidos (38 artigos). Porém, se obteve texto completo e se analisou detalhadamente somente os artigos que foram considerados relevantes para a confecção do presente trabalho (29 artigos).

RESULTADOS E DISCUSSÃO

Para entender o papel do flúor, primeiramente convém esclarecer as diferenças nas seguintes nomenclaturas: flúor é o elemento químico encontrado na natureza, enquanto fluoreto é o flúor na forma iônica $\left(F^{-}\right)$, também chamado de íon flúor.

O íon flúor ou fluoreto é o responsável pelo mecanismo anticárie do elemento químico, portanto tal mecanismo somente ocorre quando o elemento flúor está na forma iônica. Apesar disso, em português é comum o usar o termo flúor para se referir ao fluoreto, que na verdade é o seu íon. O fluoreto é encontrado em muitos alimentos tais como peixes, crustáceos, sal marinho e folhas para chá, cerveja e vinho tinto, dentre outros alimentos, mas sua principal fonte natural é a água potável ${ }^{5}$, com concentrações variáveis entre regiões.

A maneira utilizada para medir a concentração de fluoreto costuma ser a técnica do eletrodo seletivo de íons fluoreto recomendado por $\mathrm{Grøn}^{6}$, mediante p.p.m. (partes por milhão) que é a proporção de soluto em $10^{6}$ partes de solução. Desta forma, por exemplo, a água com 1 p.p.m. $F$ contém $1 \mathrm{mg} \mathrm{F}$ em 1 litro de água, ou $1 \mathrm{mg} \mathrm{F}$ em $1000 \mathrm{~g}$ de água, que é o mesmo que $1 \mathrm{mg} \mathrm{F}$ em 1000000 $\mathrm{mg}$ de água (1 parte de soluto / $10^{6}$ partes de solução).

De todas as formas, o uso de fluoretos deve ser racional e cauteloso, uma vez que pode trazer efeitos benéficos ou deletérios às dentições. Enfatizando que o efeito benéfico do fluoreto contra a cárie ocorre em função da ação tópica sobre os dentes que já irromperam, enquanto o efeito deletério no germe dentário se dá devido à ingestão e absorção sistêmica de quantidades excessivas de fluoreto em altas concentrações, provenientes de diversas fontes no período de desenvolvimento dentário (odontogênese), que vai desde antes do nascimento até os oito anos de idade, podendo desencadear um problema de saúde bucal conhecido como fluorose dentária ${ }^{4}$.

- Fluorose dentária

A fluorose dentária consiste na hipomineralização do esmalte e/ ou dentina, geralmente bilateral e simétrica, afetando com especial frequência os premolares e os segundos molares seguidos dos incisivos superiores ${ }^{7}$.

Há um grande risco de fluorose em pacientes que tenham ingestão de altas doses do íon flúor no período de formação dos elementos dentários (gestação e infância), sendo que a ingestão mais observada se deve ao uso indevido de dentifrícios fluoretados e ao consumo de água potável em regiões onde a mesma contém altas concentrações naturais de fluoreto ${ }^{3}$.

Dependendo do grau de exposição ao fluoreto, observam-se distintos graus de severidade da fluorose ${ }^{8}$. A severidade e a distribuição desse problema de saúde pública estão relacionadas com, além da quantidade da ingestão de fluoreto, com o período que a exposição acentuada ao fluoreto ocorreu e com - estágio que a amelogênese estava quando houve a ingestão ${ }^{9}$. Altos teores residuais de fluoreto trazem altos índices de fluorose dentária em crianças, comprovados em variadas localidades ${ }^{10}$.

- Índice de Dean 
A Organização Mundial da Saúde - OMS recomenda 0 registro da prevalência e severidade da fluorose aos 12 anos de idade, para permitir uma estandarização com fins de comparação entre populações. Para tal registro, pode-se contar com diferentes índices, mas o mais utilizado continua sendo 0 índice recomendado pela $\mathrm{OMS}^{11}$, mediante a classificação de Dean ${ }^{12}$ desenvolvida em 1936, que utiliza os seguintes códigos para classificar a fluorose de acordo com suas características clínicas:

$\checkmark 0=$ Normal. Superfície de esmalte lisa e brilhante, de coloração branco/creme;

$\checkmark 1$ = Discutível. Alterações na translucidez do esmalte, com pontos brancos pequenos ou manchas dispersas;

$\checkmark 2$ = Muito leve. Opacidades pequenas, brancas como papel, irregulares e disseminadas, afetando menos de $25 \%$ da face vestibular do dente;

$\checkmark 3=$ Leve. Opacidades afetando entre de 25\% e $50 \%$ da face vestibular do dente;

$\checkmark 4=$ Moderada. Esmalte mostrando um desgaste marcado, com erosão importante, podendo apresentar manchas escuras (cor marrom) salpicadas;

$\checkmark 5=$ Grave, intensa ou severa. Esmalte muito afetado e desgastado, apresentando hipoplasia de esmalte, corrosão, extensas manchas escuras e possível alteração da morfologia do dente;

$\checkmark 8$ = Excluída;

$\checkmark 9=$ Não registrada.

- Índice de Thylstrup-Fejerskov

O Índice Thylstrup-Fejerskov (TF) é um método mais recente de classificação da fluorose dentária aos 12 anos (1978), em concordância com as características clínicas e histopatológicas. A técnica tem início com a secagem prévia das superfícies dos elementos dentários com ar comprimido ou gaze, seguida por inspeção tátil visual com espelho bucal e sonda recomendada pela OMS. Quanto maior a severidade da fluorose, maior a numeração que o dente recebe. Os escores de 1 a 4 são dados quando existe aumento nos níveis da opacidade do esmalte, porém sem perda da estrutura de esmalte; enquanto os escores de 5 a 9 representam a perda do tecido mineralizado ${ }^{8,13}$. $O$ índice TF apresenta os seguintes escores:

$\checkmark 0=$ Após a secagem da superfície a translucidez normal do esmalte brilhante permanece;

$\checkmark 1$ = Pode-se ver linhas brancas cruzando a superfície dentária e uma leve cobertura parecendo neve nas cúspides e/ou margens incisais e cervicais; $\checkmark 2$ = As linhas brancas e opacas são mais pronunciadas e frequentemente se fundem para formar pequenas áreas nebulosas dispersas na superfície, sendo comum a cobertura parecendo neve nas pontas de cúspide e margens incisais;

$\checkmark 3=$ Ocorre a fusão das linhas brancas e opacas e as áreas nebulosas de opacidade aparecem em muitas partes, mas as linhas brancas ainda podem ser vistas entre as áreas nebulosas;

$\checkmark 4$ = Toda a superfície dentária exibe uma opacidade marcante com aspecto de giz branco;

$\checkmark 5=$ Toda a superfície aparece opaca e existem fissuras arredondadas com menos de $2 \mathrm{~mm}$ de diâmetro;

$\checkmark 6=$ As pequenas fissuras podem ser vistas fundindo-se no esmalte opaco formando faixas com menos de $2 \mathrm{~mm}$ de altura. Há partes em que o esmalte vestibular e das cúspides está deslaminado;

$\checkmark 7$ = Há perda do esmalte mais externo com áreas irregulares e menos da metade da superfície do esmalte envolvida onde 0 esmalte intacto remanescente está opaco;

$\checkmark 8$ = Há perda do esmalte mais externo envolvendo mais da sua metade, onde o esmalte intacto remanescente está opaco.

$\checkmark 9=$ Perda da maior parte do esmalte externo resultando na mudança anatômica do dente. Geralmente observa-se uma margem cervical de esmalte opaco.

- Prevalência da fluorose

A prevalência da fluorose segue um padrão inverso ao da cárie dentária por todo o mundo e pode ser constatada tanto em comunidades que tem programas de fluoretação das águas de abastecimento público (que é um método ou intervenção com fluoreto baseado na comunidade, nível populacional) tanto em comunidades que não tem e, portanto, consomem água natural de poços e nascentes. Neste último caso, considera-se ideal que referidas comunidades disponham de políticas públicas para detecção e inutilização de focos de fluoreto natural excessivo na água, encontrando alternativas ${ }^{9}$.

De acordo com os dados originais de Dean et al. $^{8}$, a ingestão de uma dose diária de $0.02 \mathrm{mg} F$ por $\mathrm{kg}$ de peso corporal em uma criança menor de 5 anos pode resultar em uma prevalência de cerca de $40-50 \%$ de fluorose de variadas pontuações de seu índice.

Outros autores como Bermúdez et al. ${ }^{14}$, consideram que incluso a metade da dose proposta por Dean pode ocasionar certos graus de fluorose dentária. Concretamente, a fluorose 
se produz durante a fase pré-irruptiva, quando se ingere de maneira habitual doses diárias superiores a $0.1 \mathrm{mg}$ de $\mathrm{F}$ por $\mathrm{kg}$ de peso corporal em lactantes ou superiores a $2 \mathrm{mg}$ de $\mathrm{F}$ por dia a partir dos 3 anos de idade ${ }^{14}$.

A conclusão do trabalho de Dean et al. ${ }^{12,15}$ afirmam que apareceria uma fluorose aceitável com a ingestão de 1-1.2 mg F por 1 litro de água (p.p.m. F). Porém, no estudo de Heller et al. ${ }^{16}$, se estabeleceu o nível mais seguro de 0.7 p.p.m. F. Para todos os efeitos, se considera altos níveis de fluoreto os valores maiores que 1 p.p.m. $F$ (mais que $1 \mathrm{mg}$ de $F$ por 1 litro de água) ${ }^{16}$.

Em algumas regiões da África, como por exemplo na região do Saara, a população está exposta a altos níveis de fluoreto na água potável [ $>2$ p.p.m. F ( $>2 \mathrm{mg}$ de $\mathrm{F}$ por 1 litro de água)]. Especificamente ao sul da Argélia, se diagnosticaram $95.8 \%$ de crianças com fluorose aos 12 anos, onde a concentração do íon flúor na água de consumo se apresenta entre 1.762.03 p.p.m. $\mathrm{F}^{17}$.

Em algumas regiões da Síria encontraram concentrações de fluoreto na água, ao redor de 0.8-1.9 p.p.m. F, fato que, segundo Beiruti et al. ${ }^{18}$, justifica os $15 \%$ de crianças de 12 anos examinadas no seu estudo de 2004 apresentarem fluorose.

Curiosamente, no estudo feito por Riatto et al. ${ }^{19}$ com crianças de 12 anos alojadas em um centro de refugiados no norte da África, não se apresentou nenhum caso de fluorose dentária. Este fato pôde ser atribuído à procedência síria destas crianças, pois apesar de existirem casos de fluorose na Síria (15\%), os níveis não são tão alarmantes se comparados com os da população da África Saariana (95.8\%).

Burkina-Faso é um país sem saída para o mar localizado na África ocidental, que contém concentrações de fluoreto na água potável muito variáveis dependendo da região. Assim, enquanto no estudo de Varenne et al. $^{20}$, relataram uma média baixa de 0.10 p.p.m. $F$ (0.0024-0.27 p.p.m. F), Mazza et al. ${ }^{21}$, relataram concentrações altas de fluoreto de $1.7 \pm 0.6$ p.p.m. $\mathrm{F}$ e, consequentemente, prevalência de fluorose também elevada naquela região ( $91 \%$ aos 12 anos, sendo $15 \%$ moderada a severa).

$\mathrm{Na}$ Tunísia, local aonde a concentração de fluoreto na água chega a 7 p.p.m. de íon flúor, a fluorose é considerada endêmica em algumas regiões. Em estudos realizados por Maatouk et al. ${ }^{22}$ e Abid et al. $^{23}$ observaram-se fluorose em $58 \%$ e $28 \%$ das crianças de 12 anos examinadas, respectivamente.
Em um estudo de Wondwossen et al. ${ }^{13}$ com crianças de duas regiões da Etiópia com distintas concentrações de fluoreto na água potável de 0.3-2.2 p.p.m. e 10-14 p.p.m. foram diagnosticadas $91.8 \%$ e $100 \%$ de fluorose, respectivamente. Em outro estudo realizado no mesmo ano e em paralelo à Pesquisa de Saúde Bucal da Espanha $2010^{24}$, com amostras de crianças espanholas e de crianças estrangeiras residentes na Espanha ${ }^{25}$, diagnosticaram prevalências de fluorose dentária no grupo de 12 anos de $22.9 \%$ e de $29.8 \%$, respectivamente. Vale acrescentar que a concentração de fluoreto na água de consumo na maioria das populações espanholas estudadas por Vitoria et al. ${ }^{26}$, apresentaram valores menores de 0.3 p.p.m. F.

$\mathrm{Na}$ última pesquisa de saúde bucal de âmbito nacional no Brasil, realizada pelo Ministério da Saúde no ano de $2010^{27}$, observou-se $16.7 \%$ das crianças de 12 anos apresentando algum tipo de fluorose dentária. Das crianças que foram diagnosticadas sem fluorose (83.3\%), 8.5\% foram enquadradas como discutível. Os indivíduos com fluorose muito leve corresponderam a $10.8 \%$, enquanto a fluorose leve ocorreu em $4.3 \%$ da amostra e, finalmente, o grau moderado de fluorose foi diagnosticado em $0.8 \%$. Não houve diagnóstico de fluorose dentária grave $(0 \%)$.

Vale mencionar a dificuldade de encontrar um padrão da quantidade de p. p. m. $F$ nas águas naturais brasileiras, fato que pode ser atribuído às dimensões continentais do Brasil. Porém no estudo com águas minerais disponíveis no Rio de Janeiro feito por Paixão et al. ${ }^{28}$, os resultados mostraram que a média da quantidade de fluoretos foi de 0.29 p.p.m. F com intervalo entre 0.10 e 0.74 p.p.m.

Atualmente, devido ao crescente potencial migratório mundial, o monitoramento da fluorose dentária em um país tem sua importância para comprovar se as cifras internas estão estabilizadas.

$\mathrm{Na}$ Figura 1, podem-se ver as concentrações médias de fluoreto na água de consumo e a prevalência de fluorose aos 12 anos diversos países ${ }^{5,8,17-19,21-28}$.

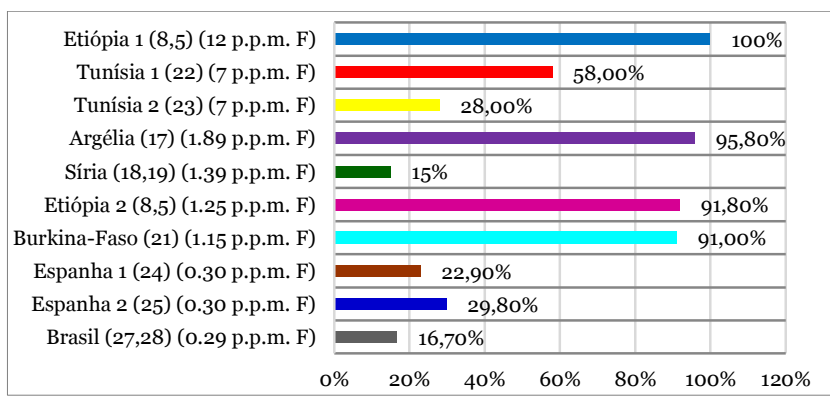

Figura 1. Prevalência de fluorose aos 12 anos nos países estudados, com base nas concentrações médias de fluoreto na água potável. 
CONCLUSÃO

A prevalência e a severidade da fluorose dentária geralmente são maiores nas populações que vivem em regiões com altas concentrações de fluoreto na água natural potável, visto que é considerada a principal fonte natural de íon flúor em muitas comunidades.

\section{REFERÊNCIAS}

1. Petersen PE, Lennon MA. Effective use of fluorides for the prevention of dental caries in the 21st century: the WHO approach. Community Dent Oral Epidemiol. 2004;32(5):319-21.

2. Leal SD, De Carvalho FS, De Carvalho CAP. Conhecimento de alunos do Curso de Odontologia sobre o uso racional do flúor. Rev Odontol da UNESP. 2015;44(1):51-8.

3. Ferreira RGLA, Bógus CM, Marques RA de A, Menezes LMB de, Narvai PC. Fluoretação das águas de abastecimento público no Brasil: o olhar de lideranças de saúde. Cad Saude Publica. 2014;30(9):1884-90.

4. Horowitz HS. Fluoride and enamel defects. Adv Dent Res. 1989;3(2):143-46.

5. Kebede A, Retta N, Abuye C, Whiting SJ, Kassaw M, Zeru $T$ et al. Dietary fluoride intake and associated skeletal and dental fluorosis in school age children in rural Ethiopian Rift Valley. Int J Environ Res Public Health. 2016;13(8):1-10.

6. Grøn P, McCann HG, Brudevold F. The direct determination of fluoride in human saliva by a fluoride electrode. Fluoride levels in parotid saliva after ingestion of single doses of sodium fluoride. Arch Oral Biol. 1968;13(2):203-13.

7. Benazzi AS, Da Silva RP, De Meneghim M, Ambrosano GM, Pereira AC. Dental caries and fluorosis prevalence and their relationship with socioeconomic and behavioural variables among 12-year-old schoolchildren. Oral Health Prev Dent. 2012;10(1):65-73.

8. Fejerskov O, Larsen MJ, Richards A, Baelum V. Dental tissue effects of fluoride. Adv Dent Res. 1994;8(1):15-31.

9. de Carvalho RB, Medeiros UV, dos Santos KT, Pacheco Filho AC. Influência de diferentes concentrações de flúor na água em indicadores epidemiológicos de saúde/doença bucal [Influence of different concentrations of fluoride in the water on epidemiologic indicators of oral health/disease]. Cien Saude Colet. 2011; 16(8):3509-18.

10. Costa $S$ de $M$. Cárie dentária e fluorose endêmica em distritos rurais de Minas Gerais, Brasil. Rev Bras Epidemiol. 2013;16(4): 1021-28.

11. Organização Mundial da Saúde. OMS. Levantamentos em Saúde Bucal: Métodos Básicos. 5. ed. São Paulo: Faculdade de Odontologia da USP; 2013.
12. Dean HT. The investigation of physiological effects by the epidemiological method. In: Moulton FR, editor. Fluorine and dental health. Washington (DC): Am Assoc Adv Sci; 1942. p.23-31.

13. Wondwossen $\mathrm{F}$, Åstrøm AN, Bjorvatn $\mathrm{K}$, Bårdsen $\mathrm{A}$. The relationship between dental caries and dental fluorosis in areas with moderate- and high-fluoride drinking water in Ethiopia. Community Dent Oral Epidemiol. 2004;32(5):337-44.

14. Bermúdez JM, Peraferrer GR, Català AG. ¿Debemos administrar suplementos orales de flúor a los lactantes? An Esp Pediatr. 1996;45(3):236-41.

15. Dean HT, Jay F, Arnold FA, Elvove E. Domestic water and dental caries: ii. a study of 2,832 white children, aged 12-14 years, of 8 suburban chicago communities, including lactobacillus acidophilus studies of 1,761 children. Pub Health Rep. 1941;56:761-92.

16. Heller KE, Eklund SA, Burt BA. Dental caries and dental fluorosis at varying water fluoride concentrations. J Public Health Dent. 1997; 57(3):136-43.

17. Almerich-Silla JM, Montiel-Company JM, RuizMiravet A. Caries and dental fluorosis in a western Saharan population of refugee children. Eur J Oral Sci. 2008;116(6):512-17.

18. Beiruti N, van Palenstein Helderman WH. Oral health in Syria. Int Dent J. 2004;54 (6 Suppl 1):383-88.

19. Riatto SG, Montero J, Pérez DR, CastañoSéiquer A, Dib A. Oral Health Status of Syrian Children in the Refugee Center of Melilla, Spain. Int J Dent. 2018;2018:2637508.

20. Varenne B, Petersen PE, Ouattara S. Oral health status of children and adults in urban and rural areas of Burkina Faso, Africa. Int Dent J. 2004;54(2):83-9.

21. Mazza C, Strohmenger L, Campus G, Cagetti MG, Caruso F, Petersen PE. Oral health status of children living in gorom-gorom, oudalan district, burkina faso. Int J Dent. 2010; 2010:597251.

22. Maatouk F, Jmour B, Ghedira K, Argoubi K, Abid A. Dental fluorosis in Kairouan, Tunisia. Dent News (Lond). 1998;1:17-9.

23. Abid A. Oral health in Tunisia. Int Dent J. 2004; 54(6 Suppl 1):389-94.

24. Llodra-Calvo JC. Encuesta de Salud Oral en España 2010. RCOE. 2012;17(1):13-41.

25. Llodra-Calvo JC. Influencia del origen de nacimiento (España versus extranjero) en la Salud Oral de la población infanto-juvenil en España 2010. RCOE. 2012;17(1):47-61.

26. Vitória I, Maraver F, Almerich-Silla JM. Flúor en aguas de consumo público españolas y prevención de la caries dental. Gac Sanit. 2014;28(3):253-59. 
27. Brasil. Ministério da Saúde. Secretaria de Vigilância em Saúde. SB Brasil 2010: Pesquisa Nacional de Saúde Bucal. Resolução no 196/96 de 7 de Janeiro de 2010. Editora MS. 2012. $116 \mathrm{p}$.

28. Paixão PJ, Arthur W, Valente $S$, Roberto $P$, Camuri N, Munhoz $\mathrm{T}$, et al. Análise da concentração de fluoretos em águas minerais disponíveis no Rio de Janeiro. Arq Bras Odontol. 2013;9(1):1-6.

\section{CONFLITO DE INTERESSES}

Os autores declaram não haver conflitos de interesse

\section{AUTOR PARA CORRESPONDÊNCIA}

\section{Vitor Matheus da Silva Luna}

Rua Honorato Araújo Filho, n`88, Nordeste 2, 58200-000 Guarabira - PB, Brasil

vitormsl.gba@gmail.com

Submetido em 02/05/2020

Aceito em 23/10/2020 